\title{
Relationship between Serum Vascular Endothelial Growth Factor Levels and Stages of Diabetic Retinopathy and Other Biomarkers
}

\author{
Nakhleh E. Abu-Yaghi $\mathbb{D}^{1,2}$ Nafez M. Abu Tarboush,, ${ }^{2,3}$ Ala M. Abojaradeh, \\ Amal S. Al-Akily, ${ }^{2}$ Esra'a M. Abdo, ${ }^{2}$ and Laith O. Emoush ${ }^{2}$ \\ ${ }^{1}$ Department of Special Surgery, Ophthalmology Division, School of Medicine, The University of Jordan, Amman, Jordan \\ ${ }^{2}$ School of Medicine, The University of Jordan, Amman, Jordan \\ ${ }^{3}$ Department of Biochemistry and Physiology, School of Medicine, The University of Jordan, Amman, Jordan
}

Correspondence should be addressed to Nakhleh E. Abu-Yaghi; nakhlehabuyaghi@yahoo.com

Received 7 May 2020; Revised 4 July 2020; Accepted 11 July 2020; Published 23 July 2020

Academic Editor: Ana Raquel Santiago

Copyright (c) 2020 Nakhleh E. Abu-Yaghi et al. This is an open access article distributed under the Creative Commons Attribution License, which permits unrestricted use, distribution, and reproduction in any medium, provided the original work is properly cited.

\begin{abstract}
Aim. This study aims to measure serum vascular endothelial growth factor (VEGF) levels in a sample of Jordanian patients and to determine their relationship with the different stages of diabetic retinopathy. It also explores the correlation between VEGF concentrations and different biochemical and demographic findings. Materials and Methods. A total of 167 adults participated in the study. Participants were divided into two main categories: patients with diabetes mellitus (DM) type 2 without diabetic retinopathy $(\mathrm{DR})(N=62)$ and patients with DM type 2 affected by DR $(N=105)$. DR patients were further subclassified into nonproliferative $(N=41)$ and proliferative $(N=64)$. Basic laboratory tests were measured to correlate with VEGF levels. Irisin, a hormone linked to diabetic retinopathy was also measured and correlated with VEGF. Results. Serum VEGF was found to positively correlate with the severity of diabetic retinopathy. The means of VEGF serum concentrations were $60 \mathrm{pg} / \mathrm{mL}$ for controls, $133 \mathrm{pg} / \mathrm{mL}$ for nonproliferative DR patients, and $229 \mathrm{pg} / \mathrm{mL}$ for proliferative DR patients. We found a significant positive correlation with glycosylated hemoglobin (HbAlc), and a significant negative correlation with high-density lipoprotein (HDL) levels, age, and irisin. Conclusion. In this cohort of Jordanian diabetics, serum VEGF concentrations strongly correlated with the presence and stages of diabetic retinopathy, suggesting it as an appropriate indicator for diabetic retinopathy early detection and management in this society. VEGF levels also significantly correlated with HbAlc, HDL, and irisin levels. Further studies are encouraged to explore these relationships in other ethnic groups and with different diabetic complications.
\end{abstract}

\section{Introduction}

Diabetes mellitus (DM) type 2 is characterized by a chronic hyperglycemic state which eventually leads, if uncontrolled, to damage in specific organs including eyes, kidneys, and peripheral nerves [1]. Ophthalmic complications include diabetic retinopathy (DR), glaucoma, cataracts, corneal abnormalities, neuropathies, and iris neovascularization [2]. $\mathrm{DR}$ is the most common and feared ophthalmic complication due to DM and has become the leading cause of vision loss in working-age adults [3]. Signs of DR are present in one-third of DM type 2 patients at the time of diagnosis, and over $60 \%$ of patients with DM type 2 will develop DR after 20 years of disease onset [4].

Advanced DR occurs when fragile new blood vessels form on the surface of the retina over time [5]. These abnormal vessels can bleed or develop scar tissue causing significant loss of sight [6]. Accompanying scar tissue can contract and cause retinal detachment from underlying tissues, thus causing permanent loss of vision. DR is classified based on the growth of new vasculature in the retina into nonproliferative (NPDR) and proliferative (PDR) [6], with the latter being a more advanced stage and most commonly responsible for visual threatening complications [7]. 
Due to the high and rising rates of DR caused by DM type 2 , it is essential to develop strategies to detect and manage this complication as early as possible. One of these strategies is the search for molecules that are associated with DR. Hence, extensive research was and is currently being conducted to investigate possible molecules linked to $\operatorname{DR}[8,9]$.

Vascular endothelial growth factor (VEGF) has emerged as an important factor associated with DR development and an important prognostic indicator of the disease [10,11]. VEGF is a $45-\mathrm{kDa}$ homodimeric glycoprotein which belongs to a wide family of growth factors [12]. It is considered one of the main proteins that induce angiogenesis [13] and regulate vascular permeability by stimulating VEGFR-1 and VEGFR2 receptors [14]. In DR, VEGF is produced by retinal cells to stimulate the development of new blood vessels adjacent to hypoxic areas $[12,15]$. VEGF also promotes microaneurysm formation and has a role in increasing the permeability of the blood-retina barrier, stimulating the neoangiogenesis process in advanced DR [13, 16]. Anti-VEGFs have been developed to neutralize the intraocular VEGF of diseased eyes by intraocular injection [17]. Metformin, which is considered the first line therapy for DM type2, was found to decrease the phosphorylation of VEGFR-2 receptor, hence inhibiting the downstream pathway resulting in a decrease in the severity of neovascularization in advanced DR [18].

Few regional studies and many international studies have revealed a significant difference in serum VEGF levels between patients with DM type 2, with or without DR $[19,20]$. A meta-analysis that was conducted in 2019 showed that serum VEGF levels correlate with the existence and severity of DR, therefore suggesting that serum levels of VEGF is a reliable indicator for DR evaluation and progression [21]. However, the relationship between VEGF serum levels and stages of DR has not been significantly explored in the Arab or Middle East regions and, up to our knowledge, was never explored in the Jordanian population where diabetes is prevalent [22].

Serum VEGF has also been studied in relation to other biochemical and demographic factors, such as glycosylated hemoglobin A1c (HbA1c), high-density lipoprotein (HDL), low-density lipoprotein (LDL), body mass index (BMI), total cholesterol (TC), triglycerides (TGs), and age without conclusive results [23-25]. Recently, DR patients have been investigated for possible association with a newly discovered myokine named "irisin" [26] that has been shown to be negatively associated with DM type 2 [27, 28]. Irisin induces browning of white adipose tissue thus protecting against weight gain and insulin resistance [29]. VEGF and irisin levels have never been correlated in the medical literature before.

Here, we aim to measure serum VEGF levels in a sample of Jordanian DM type 2 patients and determine the difference in VEGF levels among different stages of DR and investigate the correlation between VEGF concentrations and different biochemical and demographic findings including irisin.

\section{Materials and Methods}

2.1. Ethical Statement. This study has been conducted according to the Declaration of Helsinki (1964) and its amendments. The study has been approved by Jordan University Hospital (JUH) Institutional Review Board (IRB). Written informed consent has been obtained from all study participants.

2.2. Data Collection. A total of 167 adults participated in this study. Sample size was determined by power analysis and sample size software with $\alpha$ of 0.05 and power of $90 \%$. Recruitment of participants and sample collection was from March to December 2016 at the Ophthalmology Clinic at JUH. Participants were divided into two main categories: patients with DM type 2 not affected by DR as a control group $(N=62)$ and patients with DM type 2 who are affected by DR $(N=105)$. DR patients were further subclassified into two categories: NPDR $(N=41)$ and PDR $(N=64)$. Whole blood samples were collected into plain tubes. Samples were centrifuged at $1,600 \times g$ for $15 \mathrm{~min}$ at $4^{\circ} \mathrm{C}$. Serum has been kept at $-80^{\circ} \mathrm{C}$ for a period less than a month.

2.3. Serum VEGF and Irisin Measurements. The quantitative measurements of VEGF in human serum samples were performed using a commercial enzyme linked immunosorbent assay (ELISA) kits for VEGF (CK-11550, SINNOWA Medical Science \& Technology, Jiangsu, China) per the manufacturer's instructions. The absorbance from each sample was measured by a spectrophotometric microplate reader at a wavelength of $450 \mathrm{~nm}$ (Synergy ${ }^{\mathrm{TM}}$ HTX MultiMode Microplate Reader, Biotek, VT, USA). The sensitivity of the VEGF assay is typically less than $1 \mathrm{pg} / \mathrm{mL}$ and the linear range of the standard is $12.5-400 \mathrm{pg} / \mathrm{mL}$. For irisin, the quantitative measurements in human serum samples have been performed using ELISA kit (EK-067-16, Phoenix Pharmaceuticals Inc., CA, USA) per manufacturer's instructions. Absorbance from each sample has been measured in duplicate by the same spectrophotometric microplate reader at wavelength of $450 \mathrm{~nm}$. The sensitivity of the assay is $6.8 \mathrm{ng} / \mathrm{mL}$ and the linear range of the standard is $6.8-96.1 \mathrm{ng} / \mathrm{mL}$.

\subsection{Other Demographic and Biochemical Findings.} Anthropometric measurements have been obtained using standard protocols and techniques. Body mass index (BMI) has been calculated as weight in kilograms divided by the square of height in meters (BMI = weight $(\mathrm{kg}) /($ height $(\mathrm{m})$ ) 2) and used as a measure of obesity. Participants with BMI $\left(\mathrm{kg} / \mathrm{m}^{2}\right)<18.5,18.5-24.5,25-29.9$, and $\geq 30$ have been considered underweight, normal, overweight, and obese, respectively. Basic laboratory tests including $\mathrm{HbA1c}, \mathrm{HDL}$, LDL, TGs, and TC have been measured and recorded at the hospital laboratories.

2.5. Statistical Analysis. GraphPad PRISM 5 Statistical software and EXCEL Microsoft Office Professional Plus 2016 were used for statistical analyses. Continuous variables were expressed as mean \pm SD. Associations of circulating VEGF levels with patients' laboratory findings and Irisin levels have been investigated. Unpaired $t$-test was used to compare two 
TABLE 1: Demographics and clinical characteristics of DM type 2 patients included in the study. The table shows the comparison of means for each demographic and clinical finding for patients not affected (no DR) and affected by DR (NPDR and PDR).

\begin{tabular}{|c|c|c|c|c|}
\hline \multirow{2}{*}{$\begin{array}{l}\text { Parameter } \\
\mathrm{N} \text { or }(\text { mean } \pm \mathrm{SD})\end{array}$} & \multicolumn{2}{|c|}{ All DM type 2 participants } & \multicolumn{2}{|c|}{ DR group } \\
\hline & No DR group & DR group & NPDR group & PDR group \\
\hline Males/females & $29 / 33$ & $64 / 41$ & $27 / 14$ & $37 / 27$ \\
\hline Age (mean in years) & $61 \pm 11.1$ & $60 \pm 10.2$ & $63 \pm 10.9$ & $58 \pm 9.6$ \\
\hline $\operatorname{BMI}\left(\mathrm{kg} / \mathrm{m}^{2}\right)$ & $29 \pm 6.3$ & $30 \pm 5.3$ & $30 \pm 5.1$ & $30 \pm 5.6$ \\
\hline HbAlc (\%) & $7.2 \pm 1.6$ & $8.1 \pm 2.0^{*}$ & $8.1 \pm 1.8$ & $8.1 \pm 1.6$ \\
\hline $\mathrm{TC}(\mathrm{mg} / \mathrm{dL})$ & $165 \pm 45.8$ & $178 \pm 71.4$ & $165 \pm 54.4$ & $186 \pm 79.2$ \\
\hline TGs $(\mathrm{mg} / \mathrm{dL})$ & $178 \pm 118.5$ & $181 \pm 112.2$ & $167 \pm 108.8$ & $190 \pm 112$ \\
\hline $\mathrm{HDL}(\mathrm{mg} / \mathrm{dL})$ & $44 \pm 13.4$ & $40 \pm 38.8$ & $39 \pm 20.5$ & $40 \pm 47.2$ \\
\hline $\mathrm{LDL}(\mathrm{mg} / \mathrm{dL})$ & $101 \pm 37.1$ & $106 \pm 49.0$ & $96 \pm 40.3$ & $112 \pm 52.8$ \\
\hline Irisin $(\mathrm{ng} / \mathrm{mL})$ & $174 \pm 27.7$ & $99 \pm 27.5^{*}$ & $114 \pm 22.4$ & $90 \pm 25.6$ \\
\hline
\end{tabular}

${ }^{*} p$ values $<0.05$. DR: diabetic retinopathy. NPDR: nonproliferative diabetic retinopathy. PDR: proliferative diabetic retinopathy. BMI: body mass index. HbA1c: glycosylated hemoglobin. TC: total cholesterol. TGs: triglycerides. HDL: high-density lipoprotein. LDL: low-density lipoprotein.

groups, one way singly analysis of variance (ANOVA) was used to compare more than two groups, and spearman correlation analysis was used to investigate associations. Probabilities of less than 0.05 were considered statistically significant.

\section{Results}

3.1. Demographic and Clinical Parameters. The demographic and clinical parameters of DR patients and controls are displayed in Table 1. There were 93 males and 74 females recruited in the study. Sixty-two patients had DM type 2 without DR and served as controls, while 105 patients had DM type 2 with DR including NPDR and PDR. DR patients were within the age group of controls. DR patients demonstrated higher and lower significant levels of HbAlc and irisin, respectively. Other clinical parameters were not significantly different between both groups.

3.2. VEGF Analysis of DR Patients and Controls. One hundred and sixty-seven blood samples were analyzed by ELISA for VEGF concentration (sixty-two samples of patients without DR, and 105 samples of patients with DR involvement). The means of VEGF serum concentrations for controls $(N=62)$ and DR participants $(N=105)$ were $60 \pm 17.4 \mathrm{pg} / \mathrm{mL}$ and $192 \pm 66.3 \mathrm{pg} / \mathrm{mL}$, respectively. VEGF results were further analyzed for DR patients per their classification. NPDR patients $(N=41)$ had a mean of $(133 \pm 36.5 \mathrm{pg} / \mathrm{mL})$, while PDR patients $(N=64)$ had a mean of $(229 \pm 52.0 \mathrm{pg} / \mathrm{mL})$ (Figure 1). One-way single analysis of variance (ANOVA) revealed a statistically significant difference among the three groups $(p<0.001)$. Unpaired $t$-test demonstrated statistically significant differences among each two groups $(p<0.001)$.

VEGF: vascular endothelial growth factor. DR: diabetic retinopathy. PDR: proliferative diabetic retinopathy. NPDR: nonproliferative diabetic retinopathy. Mean concentration of VEGF \pm SD is shown. The difference is statistically significant among the different groups of patients $(p<0.001)$. Unpaired $t$-test demonstrated statistically significant differences among each two groups $(p<0.001)$.

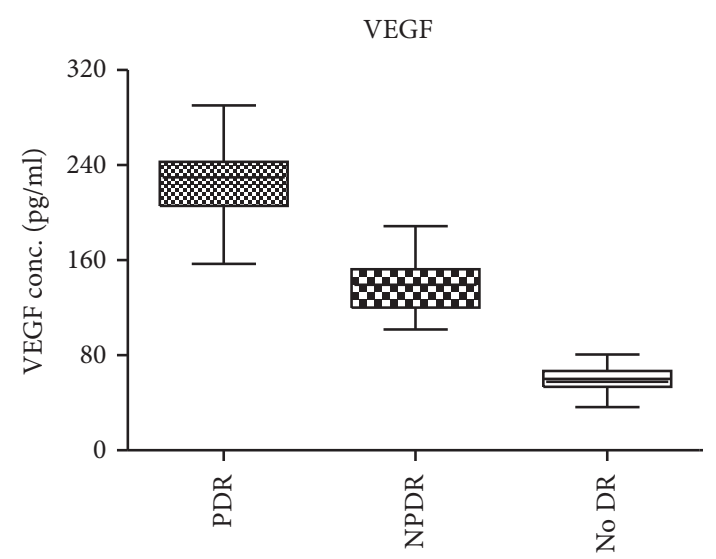

FIGURE 1: Mean concentration of VEGF hormone in serum samples of patients with DM type 2 .

3.3. VEGF Correlation to Demographic and Clinical Parameters. We also analyzed the presence of a correlation between serum VEGF concentrations and biochemical findings (HbAlc, TC, TGs, HDL, LDL, BMI, and irisin). Spearman correlation analyses revealed significant positive correlation with $\mathrm{HbAlc}(r=0.29, p=0.0002)$, and a significant negative correlation with age $(r=-0.20$, $p=0.0109)$, HDL $(r=-0.29, p=0.0007)$, and irisin $(r=-0.72, p<0.0001)$ levels. No significant correlation was found with TCs, TGs, BMI, or LDL levels (Figure 2).

\section{Discussion}

VEGF is a molecule that promotes angiogenesis and plays a critical role in the pathogenesis of DR [12, 14]. It is also one of the major indicators being studied for DR monitoring and its suppression in ocular tissues is a major target for DR treatment [17]. Studies show that VEGF serum levels in patients with DM type 2 are higher than healthy controls [30]. When injecting VEGF into animal eyes, changes similar to patients with DM type 2 or ischemic retinopathy were seen such as vessel tortuosity, retinal edema, intraretinal hemorrhages, and vascular proliferation [31]. It was also found that iris neovascularization was prevented upon inhibition of VEGF [32]. Studies on the vitreous and 


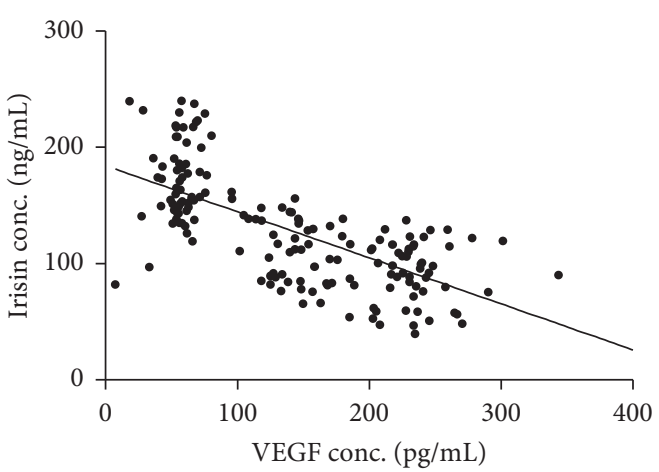

(a)

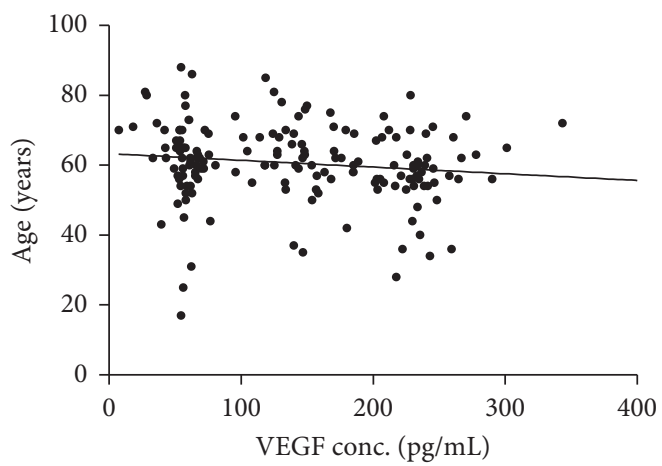

(c)

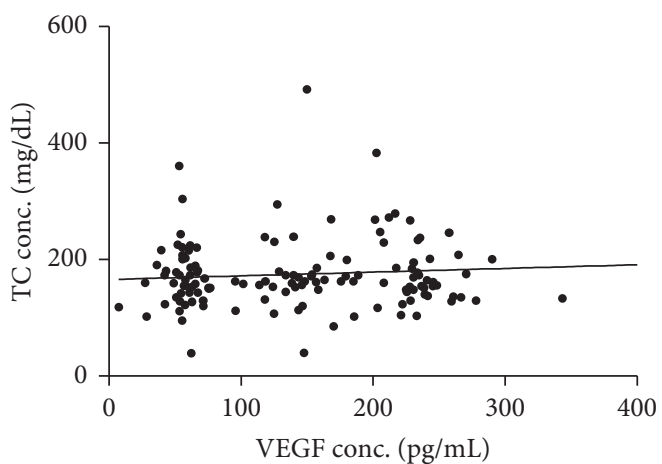

(e)

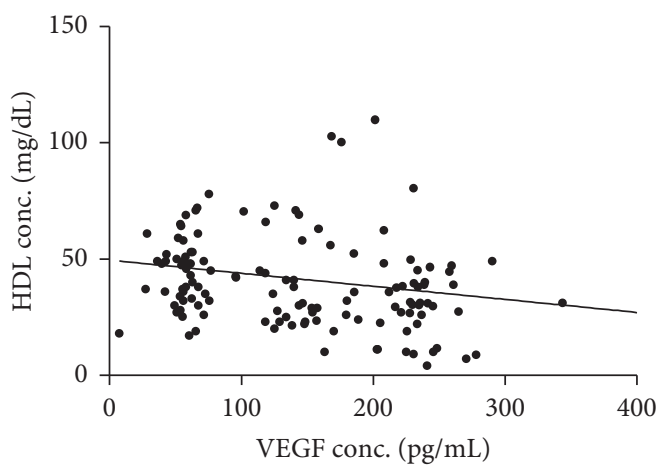

(g)

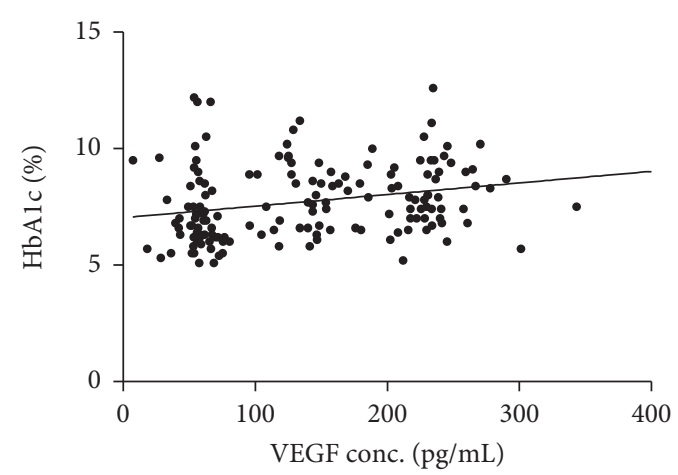

(b)

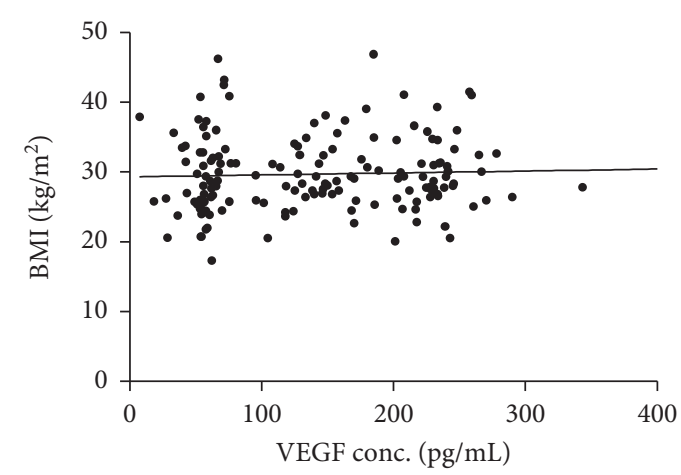

(d)

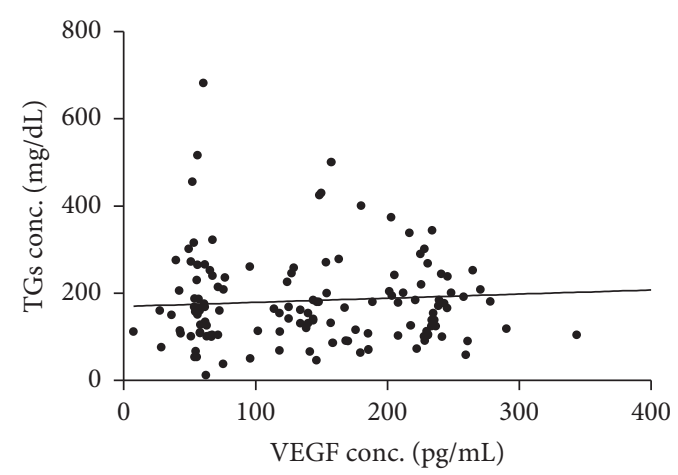

(f)

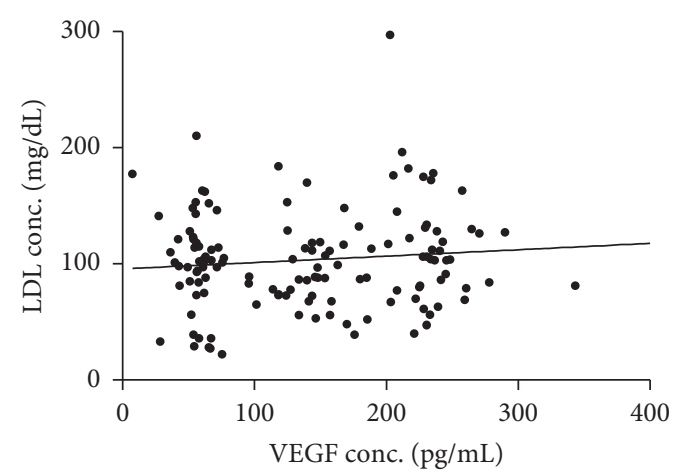

(h)

Figure 2: Continued. 


\begin{tabular}{lcccccccc}
\hline Parameter & Irisin & HbAlc & Age & BMI & TC & TGs & HDL & LDL \\
\hline$r$ & -0.72 & 0.29 & -0.2 & 0.11 & 0.02 & 0.03 & -0.29 & 0.05 \\
$p$ & $<0.0001$ & $<0.001$ & 0.01 & 0.19 & 0.83 & 0.74 & $<0.001$ & 0.57 \\
\hline
\end{tabular}

(i)

FIGURE 2: Correlation of VEGF serum level to the different clinical parameters tested for participants. VEGF: vascular endothelial growth factor. TC: total cholesterol. HbA1c: glycosylated hemoglobin. TGs: triglycerides. HDL: high-density lipoprotein. LDL: low-density lipoprotein. BMI: body mass index. $r$ indicates spearman correlation coefficient and values of $p$ less than 0.05 were considered significant.

fibrovascular tissues of human eyes have shown higher levels of VEGF in eyes affected by PDR compared to NPDR affected eyes $[33,34]$.

Studies in the region considering VEGF levels are scarce. Two studies conducted in Egypt revealed a possible association between serum VEGF levels and diabetic retinal complications including a significant difference in serum VEGF levels between PDR and NPDR which lie in accordance with results presented here $[25,35]$. Other reports in the Middle East including Saudi Arabia, Iraq, Iran, and Turkey [36-39] have only studied the relationship of VEGF levels and diabetes without differentiating between proliferative and nonproliferative DR. However, results obtained from those studies were also in line with results presented here where VEGF is higher in DM type 2 patients with DR compared to no-DR patients. Internationally, in spite of the conflicting results from different projects [40-43], most studies indicate a higher VEGF serum level in DM type 2 patients affected by DR. Nevertheless, variations in VEGF serum levels exist either regionally or internationally.

Our results demonstrate that serum VEGF levels were significantly higher in DM type 2 patients with DR compared to those with no DR. Furthermore, we found significantly higher levels of serum VEGF in patients with PDR compared to those with NPDR. These results are mirrored by reports from other parts of the world [21, 28, 43, 44]. Thus, consideration of VEGF levels as a suitable tool for risk assessment in DM type 2 patients regarding microangiopathic complications is an interesting concept despite the variation in VEGF levels amongst studies, which may reflect inherent demographic susceptibility.

This variation has been studied extensively in a recent meta-analysis that was conducted in 2019 and reviewed 29 studies with a total of 1805 DR (NPDR or PDR) patients and 1699 DM patients without DR [21]. The analysis had high heterogeneity among studies observed, but subgroup analyses and meta-regression analyses were used to adjust for potential confounders. They concluded that serum VEGF levels correlate with the existence and severity of DR, therefore suggesting that the serum VEGF level is a reliable indicator for DR evaluation and progression. Considering the VEGF level variation, the authors suggested that study location, design, and publication year of study may explain the heterogeneity in the different levels for serum VEGF among different studies [21]. We believe that our results add a much needed baseline pertaining to our community.
This study also uncovers a statistically significant correlation between VEGF levels and different biomarkers such as $\mathrm{HbAlc}, \mathrm{HDL}$, and Age. We found a significant positive correlation with $\mathrm{HbAlc}$, a finding reported in previous studies where $\mathrm{HbAlc}$ was significantly correlated with the severity of DR [23]. This is extremely important for DM type 2 patients and should be signified by general practitioners, ophthalmologists, and other specialists dealing with diabetic patients. Serum VEGF had a negative correlation with HDL levels and age in our cohort. The same results regarding HDL were found in previous studies [24]. Considering age, controversial results in correlation to VEGF levels have been published and this can be explained by the age range of the population under study and the distribution of participants to the different disease progression groups [25].

Regarding irisin, we found a negative relationship between levels of VEGF and this myokine. Irisin is a hormone which has recently been investigated as a potential biomarker for DR and was found to have lower concentrations in patients with DR compared to DM type 2 without retinopathy $[26,28]$. Irisin has also been found to behave as an anti-inflammatory agent that protects against DR through modulating the inflammatory molecule interleukin-17A [45]. Further, irisin was found to be lower in DM type 2 patients compared to healthy controls and decreases in level as the disease progresses with respect to ophthalmic complications (NPDR versus PDR) [26, 28]. VEGF level increases as retinopathy pathologically progresses; thus, it is rational for VEGF to be in negative correlation to irisin. The relationship between VEGF and irisin has never been examined in previous studies which makes this result vital for future studies.

The present study has the intrinsic limitation of being a case-control study where associations and differences are concluded, but causation is not investigated. Association of VEGF with other diabetic complications has not been explored in this project. Such relationships are expected to be intriguing as well. Also, measuring VEGF levels in samples of DM type 2 patients with and without DR involvement using multiple ELISA kits might explain the variation in serum VEGF levels in the literature and should be explored as a future direction. Up to our knowledge, this is the first study to document serum VEGF levels in the Jordanian population and establish a link with DR in such a cohort. Also, this is the first attempt to explore the relationship between VEGF and irisin. 


\section{Conclusion}

We conclude that, in this cohort of Jordanian diabetics, serum VEGF levels strongly correlated with the presence and stages of DR, showcasing this biomarker as an appropriate indicator for DR early detection and management in this ethnic group. Serum VEGF concentrations were also found to be statistically correlated with some biochemical (HbAlc, HDL, and irisin) and demographic findings (age). Further studies are encouraged to explore these relationships in other societies and with other diabetic complications.

\section{Data Availability}

The datasets generated and/or analyzed during the current study are available from the corresponding author upon reasonable request.

\section{Ethical Approval}

Institutional ethical approval was obtained from the IRB committee at Jordan University Hospital. This research complies with the tenets of the Declaration of Helsinki.

\section{Consent}

All enrolled participants provided written informed consent.

\section{Conflicts of Interest}

No conflicts of interest have been declared by the authors.

\section{Authors' Contributions}

All authors have equally contributed to the conception, data collection, data analysis, and writing and revision of the manuscript.

\section{Acknowledgments}

This study was funded by King Abdullah II Fund for Development (Grant no. 4/2016). The authors thank Miss. Suzan Albdour at the Biochemistry Laboratories for technical assistance. The authors also thank Dr. Laith Al Ejeilat and Dr. Rawand Abdel Wahed for their assistance in sample acquisition.

\section{References}

[1] American Diabetes Association, "Diagnosis and classification of diabetes mellitus," Diabetes Care, vol. 37, no. 1, pp. S81-S90, 2014.

[2] C. A. McCannel, "Retina-vitreous-macula," Mayo Clinic Proceedings, vol. 74, no. 10, pp. 1053-1054, 1999.

[3] D. Atlas, International Diabetes Federation: IDF Diabetes Atlas, International Diabetes Federation, Brussels, Belgium, 7th edition, 2015.

[4] J. W. Y. Yau, S. L. Rogers, R. Kawasaki et al., "Global prevalence and major risk factors of diabetic retinopathy," Diabetes Care, vol. 35, no. 3, pp. 556-564, 2012.

[5] R. N. Frank, "The galactosemic dog," Archives of Ophthalmology, vol. 113, no. 3, pp. 275-276, 1995.
[6] R. P. Crick and P. T. Khaw, A Textbook of Clinical Ophthalmology: A Practical Guide To Disorders Of the Eyes And Their Management, World Scientific Publishing Company, Singapore, Third edition, 2003.

[7] R. Singh, K. Ramasamy, C. Abraham, V. Gupta, and A. Gupta, "Diabetic retinopathy: an update," Indian Journal of Ophthalmology, vol. 56, no. 3, p. 179, 2008 May.

[8] C. Xu, Y. Wu, G. Liu, X. Liu, F. Wang, and J. Yu, "Relationship between homocysteine level and diabetic retinopathy: a systematic review and meta-analysis," Diagnostic Pathology, vol. 9, no. 1, p. 167, 2014.

[9] K. Zorena, D. Raczyńska, and K. Raczyńska, "Biomarkers in diabetic retinopathy and the therapeutic implications," Mediators of Inflammation, vol. 2013, Article ID 193604, 11 pages, 2013.

[10] A. P. Adamis, J. W. Miller, M.-T. Bernal et al., "Increased vascular endothelial growth factor levels in the vitreous of eyes with proliferative diabetic retinopathy," American Journal of Ophthalmology, vol. 118, no. 4, pp. 445-450, 1994.

[11] L. Han, L. Zhang, W. Xing et al., "The associations between VEGF gene polymorphisms and diabetic retinopathy susceptibility: a meta-analysis of 11 case-control studies," Journal of Diabetes Research, vol. 2014, Article ID 805801, 10 pages, 2014.

[12] L. P. Aiello, R. L. Avery, P. G. Arrigg et al., "Vascular endothelial growth factor in ocular fluid of patients with diabetic retinopathy and other retinal disorders," New England Journal of Medicine, vol. 331, no. 22, pp. 1480-1487, 1994.

[13] N. Ferrara, "Role of vascular endothelial growth factor in regulation of physiological angiogenesis," American Journal of Physiology-Cell Physiology, vol. 280, no. 6, pp. C1358-C1366, 2001.

[14] P. Carmeliet, "Mechanisms of angiogenesis and arteriogenesis," Nature Medicine, vol. 6, no. 4, pp. 389-395, 2000.

[15] E. Duh and L. P. Aiello, "Vascular endothelial growth factor and diabetes: the agonist versus antagonist paradox," Diabetes, vol. 48, no. 10, pp. 1899-1906, 1999.

[16] R. Simo, E. Carrasco, M. Garcia-Ramirez, and C. Hernandez, "Angiogenic and antiangiogenic factors in proliferative diabetic retinopathy," Current Diabetes Reviews, vol. 2, no. 1, pp. 71-98, 2006.

[17] T. Behl and A. Kotwani, "Exploring the various aspects of the pathological role of vascular endothelial growth factor (VEGF) in diabetic retinopathy," Pharmacological Research, vol. 99, pp. 137-148, 2015.

[18] Q. Y. Yi, G. Deng, N. Chen et al., "Metformin inhibits development of diabetic retinopathy through inducing alternative splicing of VEGF-A," American Journal of Translational Research, vol. 8, no. 9, pp. 3947-3954, 2016.

[19] X. Fan, Q. Wu, Y. Li et al., "Association of polymorphisms in the vascular endothelial growth factor gene and its serum levels with diabetic retinopathy in Chinese patients with type 2 diabetes: a cross-sectional study," Chinese Medical Journal, vol. 127, no. 4, pp. 651-657, 2014.

[20] M. Nalini, B. V. Raghavulu, A. Annapurna et al., "Correlation of various serum biomarkers with the severity of diabetic retinopathy," Diabetes \& Metabolic Syndrome: Clinical Research \& Reviews, vol. 11, pp. S451-S454, 2017.

[21] Z. Zhou, H. Ju, M. Sun, and H. Chen, "Serum vascular endothelial growth factor levels correlate with severity of retinopathy in diabetic patients: a systematic review and metaanalysis," Disease Markers, vol. 2019, Article ID 9401628, 15 pages, 2019.

[22] The World Bank, 2019, https://data.worldbank.org/indicator/ SH.STA.DIAB.ZS. 
[23] C. Zehetner, R. Kirchmair, M. Kralinger, and G. Kieselbach, "Correlation of vascular endothelial growth factor plasma levels and glycemic control in patients with diabetic retinopathy," Acta Ophthalmologica, vol. 91, no. 6, pp. e470-e473, 2013.

[24] J. Sajovic, I. Cilenšek, S. Mankoč et al., "Vascular endothelial growth factor (VEGF)-related polymorphisms rs10738760 and rs6921438 are not risk factors for proliferative diabetic retinopathy (PDR) in patients with type 2 diabetes mellitus (T2DM)," Bosnian Journal of Basic Medical Sciences, vol. 19, no. 1, pp. 94-100, 2019.

[25] R. A. Mahdy, W. M. Nada, K. M. Hadhoud, and S. A. ElTarhony, "The role of vascular endothelial growth factor in the progression of diabetic vascular complications," Eye, vol. 24, no. 10, pp. 1576-1584, 2010.

[26] N. A. Tarboush, N. E. Abu-Yaghi, L. H. Al Ejeilat, R. K. Wahed, and I. N. Jeris, "Association of irisin circulating level with diabetic retinopathy: a case-control study," Experimental and Clinical Endocrinology \& Diabetes, 2018.

[27] C. Zhang, Z. Ding, G. Lv, J. Li, P. Zhou, and J. Zhang, "Lower irisin level in patients with type 2 diabetes mellitus: a casecontrol study and meta-analysis," Journal of Diabetes, vol. 8, no. 1, pp. 56-62, 2016.

[28] W. Hu, R. Wang, J. Li, J. Zhang, and W. Wang, "Association of irisin concentrations with the presence of diabetic nephropathy and retinopathy," Annals of Clinical Biochemistry, vol. 53, no. 1, pp. 67-74, 2016.

[29] P. Boström, J. Wu, M. P. Jedrychowski et al., "A PGC1$\alpha$-dependent myokine that drives brown-fat-like development of white fat and thermogenesis," Nature, vol. 481, no. 7382, pp. 463-468, 2012.

[30] L. Guo, F. Jiang, Y.-T. Tang, M.-Y. Si, and X.-Y. Jiao, "The association of serum vascular endothelial growth factor and ferritin in diabetic microvascular disease," Diabetes Technology \& Therapeutics, vol. 16, no. 4, pp. 224-234, 2014.

[31] M. J. Tolentino, J. W. Miller, E. S. Gragoudas et al., "Intravitreous injections of vascular endothelial growth factor produce retinal ischemia and microangiopathy in an adult primate," Ophthalmology, vol. 103, no. 11, pp. 1820-1828, 1996.

[32] A. P. Adamis, D. T. Shima, M. J. Tolentino et al., "Inhibition of vascular endothelial growth factor prevents retinal ischemiaassociated iris neovascularization in a nonhuman primate," Archives of Ophthalmology, vol. 114, no. 1, pp. 66-71, 1996.

[33] S. K. Paine, L. K. Mondal, P. K. Borah, C. K. Bhattacharya, and J. Mahanta, "Pro- and antiangiogenic VEGF and its receptor status for the severity of diabetic retinopathy," Molecular Vision, vol. 23, pp. 356-363, 2017.

[34] X. Wang, G. Wang, and Y. Wang, "Intravitreous vascular endothelial growth factor and hypoxia-inducible factor 1a in patients with proliferative diabetic retinopathy," American Journal of Ophthalmology, vol. 148, no. 6, pp. 883-889, 2009.

[35] R. A. Mahdy and W. M. Nada, "Evaluation of the role of vascular endothelial growth factor in diabetic retinopathy," Ophthalmic Research, vol. 45, no. 2, pp. 87-91, 2011.

[36] F. A. Zakareia, A. A. Alderees, K. A. Al Regaiy, and F. A. Alrouq, "Correlation of electroretinography b-wave absolute latency, plasma levels of human basic fibroblast growth factor, vascular endothelial growth factor, soluble fatty acid synthase, and adrenomedullin in diabetic retinopathy," Journal of Diabetes and Its Complications, vol. 24, no. 3, pp. 179-185, 2010.

[37] A. M. Hasanain and M. Alsihlawi, "The relevance of serum level of VEGF in type 2 diabetic retinopathy," Kufa Medical Journal, vol. 15, no. 3, pp. 106-113, 2012.
[38] N. Baharivand, N. Zarghami, F. Panahi, M. Y. Dokht Ghafari, A. Mahdavi Fard, and A. Mohajeri, "Relationship between vitreous and serum vascular endothelial growth factor levels, control of diabetes and microalbuminuria in proliferative diabetic retinopathy," Clinical Ophthalmology, vol. 6, pp. 185-191, 2012.

[39] B. T. Ozturk, B. Bozkurt, H. Kerimoglu, M. Okka, U. Kamis, and K. Gunduz, "Effect of serum cytokines and VEGF levels on diabetic retinopathy and macular thickness," Molecular Vision, vol. 15, pp. 1906-14, 2009.

[40] H. B. Ju, F. X. Zhang, S. Wang et al., "Effects of fenofibrate on inflammatory cytokines in diabetic retinopathy patients," Medicine, vol. 96, no. 31, 2017.

[41] S. Choudhuri, I. H. Chowdhury, S. Das et al., "Role of NF- $\kappa$ B activation and VEGF gene polymorphisms in VEGF up regulation in non-proliferative and proliferative diabetic retinopathy," Molecular and Cellular Biochemistry, vol. 405, no. 1-2, pp. 265-279, 2015.

[42] K. Kaviarasan, M. Jithu, M. Arif Mulla et al., "Low blood and vitreal BDNF, LXA4 and altered Th1/Th2 cytokine balance are potential risk factors for diabetic retinopathy," Metabolism, vol. 64, no. 9, pp. 958-966, 2015.

[43] S. Ahuja, S. Saxena, L. Akduman, C. H. Meyer, P. Kruzliak, and V. K. Khanna, "Serum vascular endothelial growth factor is a biomolecular biomarker of severity of diabetic retinopathy," International Journal of Retina and Vitreous, vol. 5, no. 1, p. 29, 2019.

[44] J. Mesquita, J. P. Castro-de-Sousa, S. Vaz-Pereira, A. Neves, L. A. Passarinha, and C. T. Tomaz, "Evaluation of the growth factors VEGF-a and VEGF-B in the vitreous and serum of patients with macular and retinal vascular diseases," Growth Factors, vol. 36, no. 1-2, pp. 48-57, 2018.

[45] C. Wang, L. Wang, J. Liu et al., "Irisin modulates the association of interleukin-17A with the presence of non-proliferative diabetic retinopathy in patients with type 2 diabetes," Endocrine, vol. 53, no. 2, pp. 459-464, 2016. 